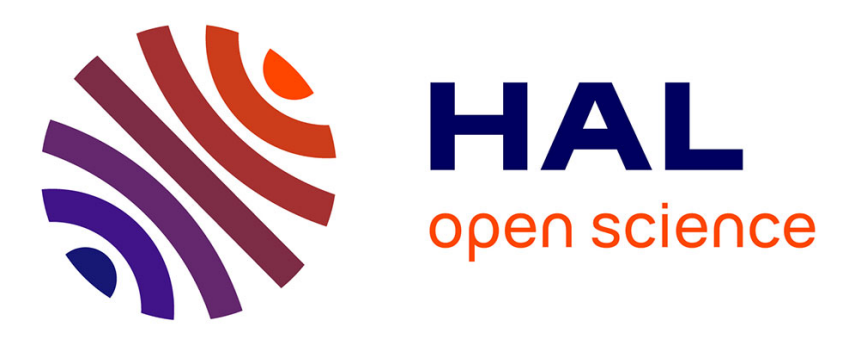

\title{
TE Band Structure for High Contrast Honeycomb Media
}

\author{
Maxence Cassier, Michael I. Weinstein
}

\section{To cite this version:}

Maxence Cassier, Michael I. Weinstein. TE Band Structure for High Contrast Honeycomb Media. Metamaterials'2020 - The 14th International Congress on Artificial Materials for Novel Wave Phenomena, Sep 2020, New-York, United States. pp.479-481, 10.1109/Metamaterials49557.2020.9284975. hal-03041931

\section{HAL Id: hal-03041931 https://hal-amu.archives-ouvertes.fr/hal-03041931}

Submitted on 5 Dec 2020

HAL is a multi-disciplinary open access archive for the deposit and dissemination of scientific research documents, whether they are published or not. The documents may come from teaching and research institutions in France or abroad, or from public or private research centers.
L'archive ouverte pluridisciplinaire HAL, est destinée au dépôt et à la diffusion de documents scientifiques de niveau recherche, publiés ou non, émanant des établissements d'enseignement et de recherche français ou étrangers, des laboratoires publics ou privés. 


\title{
TE Band Structure for High Contrast Honeycomb Media
}

\author{
Maxence Cassier $^{1}$ and Michael I. Weinstein ${ }^{2}$ \\ ${ }^{1}$ Aix Marseille Univ, CNRS, Centrale Marseille, Institut Fresnel, Faculté des Sciences - Avenue Escadrille \\ Normandie-Niémen, 13397 Marseille CEDEX, Marseille, France \\ ${ }^{2}$ Columbia University, Dept. of Applied Physics \& Applied Mathematics, and Dept. of Mathematics, 500 \\ W. 120th St., Mudd 200, MC 4701, NY 10027, New-York City, United States \\ maxence.cassier@fresnel.fr
}

\begin{abstract}
We study the band structure of transverse electric (TE) waves in a 2D medium consisting of a homogeneous bulk and a honeycomb array of higher permittivity dielectric "inclusions". In the high contrast regime and under a non-degeneracy condition, the first two dispersion surfaces touch at conical singularities, Dirac points, over the vertices of the Brillouin zone. We use analytical ideas, together with numerical simulations, to deduce detailed local information at the conical crossings as well as global behavior over the full Brillouin zone. Essential differences between this E\&M setting and the quantum model of graphene are discussed.
\end{abstract}

\section{INTRODUCTION AND MATHEMATICAL FORMULATION}

Many remarkable properties of 2D materials such as graphene and its artificial analogues are related to the presence of Dirac points in the band structure of the underlying single electron (Schrödinger) model and analogous models [2, 3]. Dirac points are energy / quasi-momentum pairs at which two adjacent dispersion surfaces touch conically. Such conical points can be explicitly displayed in the solvable tight-binding model for graphene and have been shown to exist in the continuum Schrödinger equation for generic honeycomb potentials [4]. The relationship between the strong binding (high contrast) regime and the tight binding model was studied rigorously in [5].

We explore the behavior of the TE band structure of E\&M honeycomb structures, and show that the high contrast behavior is quite different from the quantum setting. The medium has permeability $\mu_{0}>0$ and is a two-phase dielectric material: a homogeneous bulk with permittivity $\varepsilon_{0}>0$ and an array of "inclusions" of high permittivity $\varepsilon_{0} g$, where $g \gg 1$, centered on sites of a discrete honeycomb lattice. In Figure 1 the medium is partitioned into diamond-shaped cells. Each period cell has two disjoint inclusions. The physical and geometrical properties of all inclusions are the same; A (green) and B (red), indicate the two triangular sublattices that make up the honeycomb. The inclusion shape is required to be $120^{\circ}$ rotationally invariant and inversion symmetric about its center.

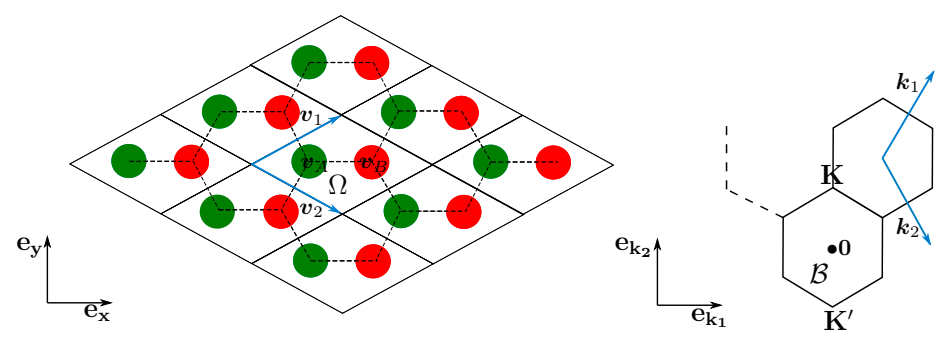

Fig. 1: Left panel: Honeycomb structure for the case where the inclusions are discs. The centers of the inclusions (vertices of the honeycomb) are located at: $\left(\boldsymbol{v}_{A}+\Lambda\right) \cup\left(\boldsymbol{v}_{B}+\Lambda\right)$, where $\Lambda=\mathbb{Z} \boldsymbol{v}_{1} \oplus \mathbb{Z} \boldsymbol{v}_{2}$ is the equilateral triangular lattice. The unit cell $\Omega$ is a diamond shape containing two inclusions centered at $v_{A}$ and $v_{B}$. Right panel: Dual lattice $\Lambda^{*}$, hexagonal Brillouin zone $\mathcal{B}$, dual basis $\left(\boldsymbol{k}_{1}, \boldsymbol{k}_{2}\right)$ and high symmetry quasimomenta $\mathbf{K}$ and $\mathbf{K}^{\prime}$.

TE modes are characterized by the triple $\left(E_{x}, E_{y}, H_{z}\right)$, where $H_{z}$ satisfies for $\omega \neq 0:-\nabla \cdot \varepsilon_{g}^{-1} \nabla H_{z}=\omega^{2} \mu_{0} H_{z}$. The transverse field, $\left(E_{x}, E_{y}\right)$, is computed explicitly from $H_{z}$. Here, $\varepsilon_{g}$ is independent of $z: \varepsilon_{g}(x, y)=\varepsilon_{0} g$ in 
all the inclusions, and $\varepsilon_{g}(x, y)=\varepsilon_{0}$ outside the inclusions. After of multiplication of the $H_{z}-$ equation by $\varepsilon_{0} g$, we obtain a spectral problem for the 2nd order self-adjoint elliptic operator $\mathbb{A}_{g}=-\nabla \cdot \sigma_{g} \nabla$ :

$$
\begin{aligned}
\mathbb{A}_{g} H_{z} & =\lambda H_{z}, \quad \text { with spectral parameter } \lambda=g\left(\frac{\omega}{c}\right)^{2}, c=\left(\mu_{0} \varepsilon_{0}\right)^{-1 / 2}, g \gg 1, \\
\text { and } \quad \sigma_{g}: & : \begin{cases}\sigma_{g}(x, y)=1, & \text { for }(x, y) \text { in the inclusions, } \\
\sigma_{g}(x, y)=g, & \text { for }(x, y) \quad \text { in the bulk. }\end{cases}
\end{aligned}
$$

Since $\mathbb{A}_{g}$ commutes with triangular lattice $(\Lambda)$ translations, its spectrum is obtained via a family of pseudoperiodic Floquet-Bloch eigenvalue problems: for each $\boldsymbol{k}=\left(k_{x}, k_{y}\right) \in \mathcal{B}$, the hexagonal Brillouin zone (Figure 1), we denote by $\lambda_{1}(\boldsymbol{k} ; g) \leq \lambda_{2}(\boldsymbol{k} ; g) \leq \ldots \lambda_{n}(\boldsymbol{k} ; g) \leq \ldots$ the sequence of eigenvalues (with multiplicities listed) for the eigenvalue problem: $\mathbb{A}_{g} H_{z}=\lambda H_{z}$ subject to $\boldsymbol{k}$-quasiperiodic boundary conditions. The graphs of the Lipschitz continuous maps $\boldsymbol{k} \mapsto \lambda_{n}(\boldsymbol{k} ; g), n \geq 1$, are called dispersion surfaces. The $L^{2}\left(\mathbb{R}^{2}\right)$ spectrum of $\mathbb{A}_{g}$ is the union of closed real intervals, that are swept out by its dispersion maps as $k$ varies over $\mathcal{B}$. The collection of all Floquet-Bloch eigenvalue / eigenfunction pairs is referred to as the band structure of $\mathbb{A}_{g}$.

Finally, the energy / quasimomentum pair $\left(\lambda_{D}, \boldsymbol{k}_{D}\right)$ is a Dirac point if there are consecutive dispersion maps $\lambda_{-}(\boldsymbol{k} ; g) \leq \lambda_{+}(\boldsymbol{k} ; g)$ which touch conically at $\left(\lambda_{D}, \boldsymbol{k}_{D}\right)$ : there exists $v_{D}>0$ such that

$$
\lambda_{ \pm}(\boldsymbol{k} ; g)=\lambda_{D} \pm v_{D}\left|\boldsymbol{k}-\boldsymbol{k}_{D}\right|\left(1+O\left(\left|\boldsymbol{k}-\boldsymbol{k}_{D}\right|\right)\right), \quad \boldsymbol{k} \text { near } \boldsymbol{k}_{D}
$$

For results on Dirac points for smooth E\&M honeycomb structures not in a high contrast regime, we refer to [7].

\section{SUMmaRY OF THE MAIN RESUltS}

We summarize our results on the band structure of $\mathbb{A}_{g}$ in the high contrast regime. We focus here on the two first dispersions surfaces $\lambda_{1}(\cdot ; g)$ and $\lambda_{2}(\cdot ; g)$. Our results generalize to higher frequency dispersion surfaces in the particular case where the inclusions are circular.

(I) High contrast asymptotic behaviour of the two first dispersion surfaces: For $g$ sufficiently large we have that:

- there exists a gap between the second $\lambda_{2}(\cdot ; g)$ and third dispersion $\lambda_{3}(\cdot ; g)$ surfaces (over the full Brillouin zone).

- As $g \rightarrow+\infty, \lambda_{1}(\cdot ; g)$ converges uniformly to the constant function equal to the first Dirichlet eigenvalue $\delta_{1}>0$ of a single inclusion, uniformly on any compact subset of $\mathcal{B}$, which excludes $\boldsymbol{k}=0$. The uniform convergence on all of $\mathcal{B}$ fails since $\lambda_{1}(\mathbf{0} ; g)=0$ for all $g>0$; constants are periodic eigenfunctions with eigenvalue zero.

- The second dispersion map $\lambda_{2}(\cdot, g)$ converges uniformly as $g \rightarrow+\infty$ to the constant function $\delta_{1}$ on $\mathcal{B}$.

(II) Existence of Dirac point for high contrasts:

Let $\lambda_{-}=\lambda_{1}(\boldsymbol{k} ; g)$ and $\lambda_{+}=\lambda_{2}(\boldsymbol{k} ; g)$. Then, for $g$ sufficiently large, the local character of the first two dispersion surfaces is described by (1) $\left(\lambda_{D}, \boldsymbol{k}_{D}\right)$, where $\boldsymbol{k}_{D}$ is any of the six vertices of $\mathcal{B}$. The constant $v_{D}$ (the "Dirac" or "Fermi velocity") is explicitly given in terms of a basis of the two dimensional subspace of degenerate Floquet Bloch modes at $\boldsymbol{k}=\boldsymbol{k}_{D}$. Under the non-degeneracy condition that $v_{D} \neq 0$, the $\left(\lambda_{D}, k_{D}\right)$ are Dirac points.

We refer to figure 2 for a numerical illustration of the results (I) and (II). The proof of result (I) makes use of the variational theory developed in [6] for high contrast elliptic PDE. Important use is made of (i) the monotonicity of the energy form: $a_{g}(u, u)=\int_{\Omega} \sigma_{g}|\nabla u|^{2} \mathrm{~d} \boldsymbol{x}$ with respect to the contrast parameter $g$, and the min-max characterization of eigenvalues of self-adjoint-operators.

(III) Asymptotic expansions of Floquet-Bloch eigenvalue and eigenfunction at the vertices of the Brillouin zone:

We obtain asymptotic expansions at any order in powers of $g^{-1}$ of: the Dirac energy for the first two bands, $\lambda_{D}(g)$, and a basis, $\left\{\Phi_{1}, \Phi_{2}\right\}$, of the two-dimensional degenerate subspace, nullspace $\left(\mathbb{A}_{g}-\lambda_{D}\right)$, associated with the Dirac point. To leading order $\lambda_{D}(g)=\delta_{1}+\mathcal{O}\left(g^{-1}\right)$, where $\delta_{1}$ is the above-mentioned first Dirichlet eigenvalue. A basis of nullspace $\left(\mathbb{A}_{g}-\lambda_{D}\right)$ consists of $\Phi_{1}(\boldsymbol{x}, g)=P_{\boldsymbol{k}_{D}, A}(\boldsymbol{x})+\mathcal{O}\left(g^{-1}\right)$ and $\Phi_{2}(\boldsymbol{x}, g)=P_{\boldsymbol{k}_{D}, B}(\boldsymbol{x})+\mathcal{O}\left(g^{-1}\right) . P_{\boldsymbol{k}_{D}, A}$ and $P_{\boldsymbol{k}_{D}, B}$ are $\boldsymbol{k}_{D}-$ pseudo-periodic superpositions of single inclusion Dirichlet states; $P_{\boldsymbol{k}_{D}, A}$ is supported on the $A$ - inclusions and $P_{\boldsymbol{k}_{D}, B}$ is supported on the $B$ - inclusions; see figure 3 . They play the role of atomic orbitals in the strong-binding Schrödinger analysis; see [5]. In contrast to ground state quantum atomic orbitals, these approximations are compactly and disjointly supported, rather than exponentially localized in the well-depth parameter. 

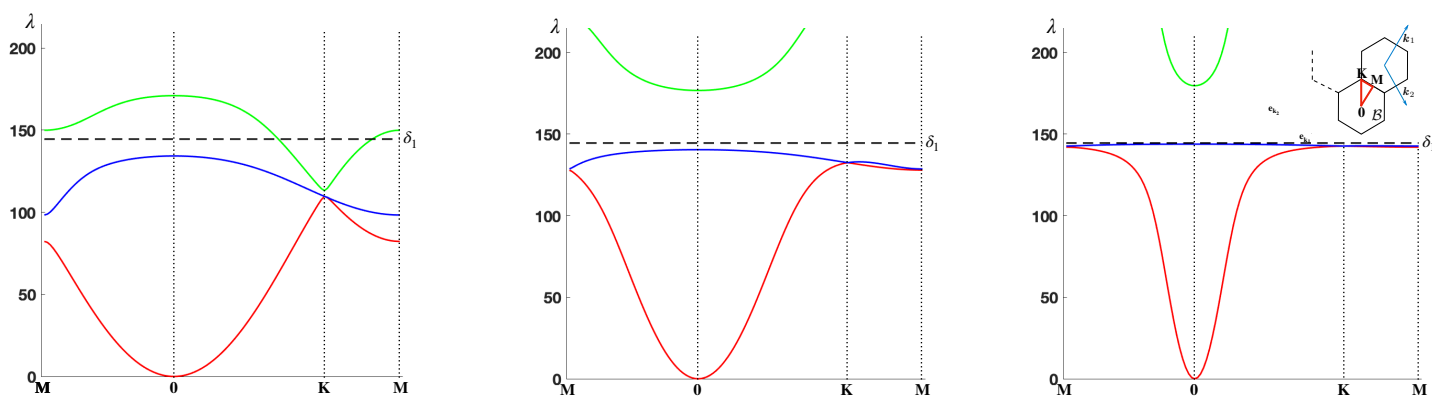

Fig. 2: $\boldsymbol{k} \mapsto \lambda_{1}(\boldsymbol{k} ; g), \lambda_{2}(\boldsymbol{k} ; g)$ and $\lambda_{3}(\boldsymbol{k} ; g)$ for two disks inclusions of radius $R=0.2$ (per cell) plotted along the contour: M-0-K-M of the reduced Brillouin zone in red (right) for $g=14$ (left), $g=35$ (center) and $g=200$ (right). The Dirac point $\boldsymbol{k}_{D}$ is located at $\mathbf{K}=\left(\mathrm{K}_{x}, \mathrm{~K}_{y}\right)=(0,4 \pi / 3)$ (center and right).

The disjointness of the supports of $\Phi_{j}(\cdot, g)$ at leading order implies $v_{D}(g)=v_{D}^{(1)} g^{-1}+\mathcal{O}\left(g^{-2}\right)$. Numerically, we observe $v_{D}^{(1)} \neq 0$. Thus, for $g \gg 1,\left(\lambda_{D}, \boldsymbol{k}_{D}\right)$ is a non-degenerate conical (Dirac) point. Note: in the quantum case the lowest two dispersion surfaces convergence uniformly on all of $\mathcal{B}$ to that those of a tight-binding model [5].

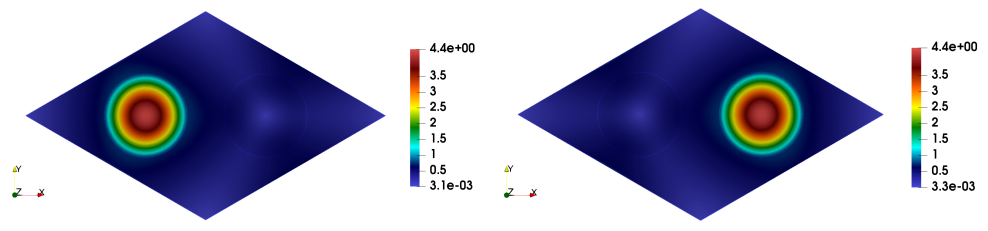

Fig. 3: $\left|\Phi_{1}(g ; \cdot)\right|$ and $\left|\Phi_{2}(g ; \cdot)\right|$ on fundamental period cell $\Omega$; contrast $g=14$; disc inclusion radius $R=0.2$.

\section{CONCLUSION}

We study the first two TE bands of a class of high contrast honeycomb dielectric media. We elucidate the convergence of the first two dispersion surfaces and give a detailed analysis of the behavior near Dirac points over the Brillouin zone vertices. We corroborate and extend our analysis with detailed numerical simulations. Finally, we point to differences in the nature of the band spectrum of high contrast dielectric media, such as photonic crystals, and their quantum analogues. An article with complete details and extensions is in preparation [1].

\section{ACKNOWLEDGEMENT}

This work was supported by US NSF-grants DMS-1412560, DMS-1620418, DMS-1908657, and Simons Foundation Math + X award \#376319 (M. I. Weinstein).

\section{REFERENCES}

[1] M. Cassier and M.I. Weinstein, "High contrast elliptic operators in honeycomb structures," in preparation.

[2] A.H. Castro Neto, F. Guinea, N.M.R. Peres, K.S. Novoselov and A.K. Geim, " The electronic properties of graphene," Rev. of Mod. Physics, vol. 81, p.109, 2009.

[3] T. Ozawa, H.M. Price, A. Amo, N. Goldman, M. Hafezi, L. Lu, M.C. Rechtsman, D. Schuster, J. Simon, O. Zilberberg and I. Carusotto, “ Topological Photonics," Rev. of Mod. Physics, vol. 9, 015006, 2019.

[4] C.L. Fefferman and M.I. Weinstein, "Honeycomb lattice potentials and Dirac points," J. Amer. Math. Soc., vol. 25, p. 1169,2012

[5] C.L. Fefferman, J.P. Lee-Thorp and M.I. Weinstein, "Honeycomb Schrödinger operators in the strong binding regime," Comm. in Pure and Appl. Math., vol. 71 (6), p. 178, 2018.

[6] R. Hempel, K. Lienau, "Spectral properties of periodic media in the large coupling limit: Properties of periodic media," Comm. PDE, vol. 25 (7-8), p. 1445, 2000.

[7] J.P. Lee-Thorp, M.I. Weinstein and Y. Zhu, "Elliptic operators with honeycomb symmetry: Dirac points, Edge States and Applications to Photonic Graphene," Arch. Ration. Mech. Anal., vol. 232 (1), p. 1, 2019. 